\title{
Analysis of the potential energy surface of $\mathrm{Ar}-\mathrm{NH}_{3}$
}

\author{
G. Chalasinski, a) S. M. Cybulski, M. M. Szczessniak, and S. Scheiner \\ Department of Chemistry and Biochemistry, Southern Illinois University, Carbondale, Illinois 62901
}

(Received 30 May 1989; accepted 8 September 1989)

\begin{abstract}
The combination of supermolecular Møller-Plesset treatment with the perturbation theory of intermolecular forces is applied in the analysis of the potential energy surface of $\mathrm{Ar}-\mathrm{NH}_{3}$. Anisotropy of the self-consistent field (SCF) potential is determined by the first-order exchange repulsion. Second-order dispersion energy, the dominating attractive contribution, is anisotropic in the reciprocal sense to the first-order exchange, i.e., minima in one nearly coincide with maxima in the other. The estimated second-order correlation correction to the exchange effect is nearly as large as a half $\Delta E^{\mathrm{SCF}}$ in the minimum and has a "smoothing" effect on the anisotropy of $\epsilon_{\mathrm{disp}}^{(20)}$. The model which combines $\Delta E^{\mathrm{SCF}}$ with dispersion energy $(\mathrm{SCF}+\mathrm{D})$ is not accurate enough to quantitatively describe both radial and angular dependence of interaction energy. Comparison is also made between $\mathrm{Ar}-\mathrm{NH}_{3}$ and $\mathrm{Ar}-\mathrm{PH}_{3}$, as well as with the Ar dimer.
\end{abstract}

\section{INTRODUCTION}

van der Waals complexes display unusual structural and dynamic properties resulting from the shapes of their potential energy surfaces. ${ }^{1}$ The weak interactions in these complexes lead to surfaces that are very flat and contain multiple minima. At present, there is no simple rationalization for the structures of such complexes. For example, Shea and Campbell ${ }^{2}$ explain the position of the Ar atom with respect to $\mathrm{F}_{2} \mathrm{CO}$, as well as in a number of other complexes, on the basis of preference of the rare gas atom for the more electropositive atoms. However, the experimentally known structure of the $\mathrm{Ar}-\mathrm{NH}_{3}$ complex cannot be predicted by this rule. As found by Klemperer and co-workers, ${ }^{3}$ the $\mathrm{Ar}-\mathrm{N}$ line is nearly perpendicular to the $C_{3}$ axis of the ammonia molecule. Recent calculations by Latajka and Scheiner confirmed the same type of structure for the $\mathrm{Ar}-\mathrm{PH}_{3}$ complex. ${ }^{4}$ These authors found that despite the fact that the bonding is largely due to correlation, the minimum energy structure is determined by the anisotropy of the self-consistent field (SCF) interaction energy. No further details as to the origin of this anisotropy were given.

The potential energy surface represents the superposition of a number of various interaction energy terms such as electrostatic, exchange, induction, dispersion, and their respective intrasystem correlation corrections. These terms each have different distance and angular dependencies. In order to explain and qualitatively predict the structures of van der Waals complexes, anisotropies of each of these terms should be examined separately. With some of them, such as those occurring within the Hartree-Fock interaction energy, the properties are quite well understood (although rigorous calculations of these terms are surprisingly scarce). However, the complexes in question are mainly bound by correlation effects whose properties are not as yet very thoroughly characterized. ${ }^{5}$ Our goal is to rationalize the experimentally known structure of the $\mathrm{Ar}-\mathrm{NH}_{3}$ complex on the

\footnotetext{
a Department of Chemistry, University of Warsaw, Pasteura 1, 02-093 Warsaw, Poland
}

basis of analysis of each interaction energy term separately and in this way devise a set of rules which could help in predicting structures of other complexes.

The Ar- $\mathrm{NH}_{3}$ complex is very attractive for such a study. First of all, the detailed molecular beam study by Klemperer et $a l^{3}{ }^{3}$ provides not only the structural features, but also some estimate of the dissociation energy. The system is small enough so that a high accuracy treatment can be applied. Finally, due to the fact that one of the subsystems is spherically symmetric, the multipole part of the electrostatic energy vanishes at the HF level, as well as at correlated levels, which simplifies certain aspects of the analysis.

In this paper we propose an approach to the study of intermolecular interactions which combines the supermolecular Møller-Plesset Perturbation Theory with the Perturbation Theory of Intermolecular Forces. The goal of this paper is to demonstrate that such combination may serve as a very powerful tool in studies of molecular complexes at quantitative levels of theory.

\section{METHOD AND DEFINITIONS}

Most $a b$ initio calculations of interaction energies with the inclusion of correlation effects are performed using the supermolecular method based on the Moller-Plesset perturbation theory (MPPT).$^{6.7}$ Such a treatment is well justified in that it properly includes intermolecular exchange effects. However, the interpretative power of this method is quite limited since it does not allow for separate analysis of individual interaction energy terms. On the other hand, the partitioning of the interaction energy into meaningful and physically interpretable terms is achieved naturally through perturbation theory of intermolecular forces which treats the intermolecular interaction as a perturbation. One example of such a theory is the intermolecular Moller-Plesset perturbation theory (IMPPT), ${ }^{8}$ which was designed by Jeziorski, Szalewicz, and collaborators in its most complete, symmetry-adapted form. ${ }^{9,10}$ Unfortunately, the latter approach has some disadvantages too, mainly with reference to the treatment of intermolecular exchange effects.

The recently established connection between MPPT 
and IMPPT helps to alleviate weaknesses of both methods. " In particular, the simultaneous application of both methods allows one to take advantage of the interpretability of IMPPT interaction energy terms within the framework of MPPT. Meanwhile, the questionable treatment of exchange terms can be avoided, the information concerning these effects extracted from MPPT which properly deals with the intersystem symmetry. By exploring such a combined treatment in application to a model system, we hope to provide a further justification for its usefulness in studies of weak intermolecular interactions.

The correlation energy was derived using the MPPT treatment ${ }^{6}$ through the full fourth order of perturbation theory employing the frozen core approximation (i.e., the first five orbitals of Ar were frozen). The latter has proven reliable in calculations of the interaction energy of the Ar dimer. ${ }^{12}$ The MPPT interaction energy corrections are derived as a difference between the values for the total energy of the dimer and the sum of the subsystem energies, derived in the basis set of the dimer in every order of perturbation theory

$$
\begin{aligned}
\Delta E^{\mathrm{SCF}} & =E_{A B}^{\mathrm{SCF}}-E_{A}^{\mathrm{SCF}}-E_{B}^{\mathrm{SCF}}, \\
\Delta E^{(n)} & =E_{A B}^{(n)}-E_{A}^{(n)}-E_{B}^{(n)}, \quad n=2,3,4 .
\end{aligned}
$$

The sum of corrections through the $n$th order will be denoted $\Delta E(n)$; thus, e.g., $\Delta E(3)$ will symbolize the sum of $\Delta E^{\mathrm{SCF}}, \Delta E^{(2)}$, and $\Delta E^{(3)}$. The interaction energy corrections of IMPPT are denoted $\epsilon^{(i j)}$, where $i$ and $j$ refer to the order with respect to the intermolecular interaction operator and the intramolecular correlation operator, respectively.

\section{A. Partitioning of $\Delta E^{\mathrm{scF}}$}

$$
\begin{aligned}
& \Delta E^{\mathrm{SCF}} \text { may be decomposed as } \\
& \Delta E^{\mathrm{SCF}}=\Delta E^{\mathrm{HL}}+\Delta E_{\text {def }}^{\mathrm{SCF}} .
\end{aligned}
$$

The Heitler-London interaction energy is defined as

$$
\begin{aligned}
\Delta E^{\mathrm{HL}}= & E_{A B}^{\mathrm{HL}}-E_{A}^{\mathrm{SCF}}-E_{B}^{\mathrm{SCF}}, \\
E_{A B}^{\mathrm{HL}}= & \left\langle\mathscr{A} \Phi_{A}^{\mathrm{SCF}} \Phi_{B}^{\mathrm{SCF}}|\mathscr{H}| \mathscr{A} \Phi_{A}^{\mathrm{SCF}} \Phi_{B}^{\mathrm{SCF}}\right\rangle / \\
& \left\langle\mathscr{A} \Phi_{A}^{\mathrm{SCF}} \Phi_{B}^{\mathrm{SCF}} \mid \Phi_{A}^{\mathrm{SCF}} \Phi_{B}^{\mathrm{SCF}}\right\rangle,
\end{aligned}
$$

where $\mathscr{A}$ denotes antisymmetrizer, $\Phi^{\mathrm{SCF}}$ denotes the SCF wave function for isolated $A$ or $B$ monomers, and $\mathscr{H}$ refers to the total Hamiltonian. $\Delta E^{\mathrm{HL}}$ may be separated into electrostatic and exchange contributions ${ }^{13}$

$$
\epsilon_{\text {elst }}^{(10)}=\left\langle\Phi_{A}^{\mathrm{SCF}} \Phi_{B}^{\mathrm{SCF}}|\boldsymbol{V}| \Phi_{A}^{\mathrm{SCF}} \Phi_{B}^{\mathrm{SCF}}\right\rangle,
$$

where $V$ denotes the interaction operator and

$$
\epsilon_{\text {exch }}^{(10)} \equiv \Delta E^{\mathrm{HL}}-\epsilon_{\text {elst }}^{(10)} \text {. }
$$

$\epsilon_{\text {exch }}^{(10)}$ so defined differs slightly from the definition of Ref. 13 by the presence of so-called "zeroth-order exchange" terms $\Delta_{F}$ and $\Delta_{W}$. As shown by Gutowski et al., ${ }^{14} \Delta_{F}$ vanishes for exact $\Phi_{A}^{\mathrm{SCF}}, \Phi_{B}^{\mathrm{SCF}}$ functions or when they are derived within the dimer centered basis set (DCBS). $\Delta_{W}$ is of the order of the fourth power of the intersystem overlap integral; it is thus small. Both $\Delta_{F}$ and $\Delta_{W}$ are of exchange type, therefore it seems legitimate to incorporate them into $\epsilon_{\mathrm{exch}}^{(10)}{ }^{14,15}$

The SCF interaction energy $\Delta E^{\mathrm{SCF}}$ differs from the Heitler-London energy by the presence of terms which are due to deformations of the subsystems' wave functions. The SCF deformation term is defined by Eq. (2):

$$
\Delta E_{\text {def }}^{\mathrm{SCF}}=\Delta E^{\mathrm{SCF}}-\Delta E^{\mathrm{HL}} .
$$

For large intersystem distances, $\Delta E_{\text {def }}^{\text {SCF }}$ may be interpreted as the induction energy. ${ }^{16}$ However, since induction evaluated with neglect of exchange effects may lead to collapsing of the electrons of one fragment into the occupied orbitals of the other fragment, ${ }^{17}$ we avoid any further partitioning of $\Delta E_{\text {def }}^{\mathrm{SCF}}$.

\section{B. Partitioning of $\Delta E^{(2)}$}

$\Delta E^{(2)}$ may be decomposed as follows ${ }^{7,11}$ : $\Delta E^{(2)}=\epsilon_{\text {disp }}^{(20)}+\epsilon_{\text {elst }}^{(12)}+$ induction correlation + exchange terms,

where $\epsilon_{\text {disp }}^{(20)}$ denotes the second-order UCHF dispersion energy as defined in Ref. 18, and $\epsilon_{\text {elst }}^{(12)}$ represents the secondorder intrasystem correlation correction to the electrostatic effect. The definition of this term was proposed by Jeziorski and collaborators $10(a)$ and recently augmented by the "response" or "orbital relaxation" terms. ${ }^{10(b), 10(c)}$

The "exchange terms" in Eq. (7) encompass the exchange counterparts of dispersion, electrostatic, and induction correlation, which are extremely difficult to calculate directly. ${ }^{7,8}$ Equation (7) provides a recipe for an indirect estimate of those effects. If induction correlation could be assumed small which is certainly the case in $\mathrm{Ar}-\mathrm{NH}_{3}$, the second-order exchange effect may be approximately evaluated as

$$
\Delta E_{\mathrm{exch}}^{(2)} \cong \Delta E^{(2)}-\epsilon_{\mathrm{disp}}^{(20)}-\epsilon_{\mathrm{elst}}^{(12)} .
$$

If additionally the $\epsilon_{\text {elst }}^{(12)}$ term is small, especially in atommolecule interactions where it has no multipole component, an even simpler approximation for the second-order exchange effect may be used

$$
\Delta E_{\mathrm{exch}}^{(2)} \cong \Delta E^{(2)}-\epsilon_{\mathrm{disp}}^{(20)} \text {. }
$$

\section{Calculations of interaction energies}

Calculations of all the supermolecular and perturbational interaction terms are performed using dimer centered basis sets (DCBS). With supermolecular interaction energies, this prescription amounts to applying the counterpoise procedure of Boys and Bernardi. ${ }^{19}$ There is strong evidence that this is the only consistent means of evaluation of interaction energy at the $\mathrm{SCF}^{20,21}$ as well as at correlated levels. ${ }^{15,22,23}$ With perturbation terms the description of subsystem wave functions in dimer basis sets has important implications. First, as mentioned earlier, the DCBS treatment leads to vanishing of the unphysical $\Delta_{F}$ term within the Heitler-London energy $\Delta E^{\mathrm{HL}}[\mathrm{Eq}$. (3) $] .^{14,15}$ Second, it also improves the description of $\epsilon_{\text {exch }}^{(10)},{ }^{14} \epsilon_{\text {ind }}^{(20)}$, and $\epsilon_{\text {disp }}^{(20)}{ }^{18}$ It may, however, deteriorate $\epsilon_{\text {elst }}^{(10)}$. Third, using DCBS consistently is absolutely necessary if individual components of interaction energy are extracted by means of subtraction as in Eqs. (6), (8), and (9). Failure to comply with this condition led Collins and Gallup to obtain unphysical repulsive deformation energies. ${ }^{21,24}$ 


\section{Basis sets}

The $[7 s, 4 p, 2 d]$ and $[7 s, 4 p, 2 d, 1 f]$ basis sets for Ar originate from the study of $(\mathrm{Ar})_{2}$ by Chalasinski et al. ${ }^{12}$ The exponents of $d$ functions are $0.84,0.174$, and for the $f$ function 0.23 (unfortunately, there is a misprint of the second $d$ and the $f$ exponents in Ref. 12). Their values are chosen to maximize the intersystem correlation effects. The energetic characteristics of both basis sets are provided in Ref. 12. The values of dipole polarizability are presented here in Table $I$.

For $\mathrm{NH}_{3}$, we used the "medium-polarized" basis sets proposed by Sadlej ${ }^{25}: \mathrm{N}[5 s, 3 p, 2 d], \mathrm{H}[3 s, 2 p]$. These basis sets have proven very reliable in calculations of intermolecular forces. ${ }^{26}$ The polarization functions in medium-polarized basis sets are chosen as the electric field derivatives of the atomic energy optimized $s p(\mathrm{~N})$ and $s(\mathrm{H})$ sets; they are contractions of four primitives. ${ }^{25}$ Selected points were calculated with the basis set further augmented by an $f$ function with exponent $0.228 .{ }^{27}$ Electric properties of $\mathrm{NH}_{3}$ in both basis sets are shown in Table I. The internal geometry of $\mathrm{NH}_{3}$ was assumed to be undistorted by the interaction: the experimental geometry with $r(\mathrm{NH})=1.01242 \AA$ and $\theta(\mathrm{HNH})=106.67^{\circ}$ was the same as in Ref. 27. The supermolecular calculations were carried out using the Gaussian 86 program. $^{31}$

\section{RESULTS}

\section{A. Anisotropy of interaction energy}

The geometrical parameters of the $\mathrm{Ar}-\mathrm{NH}_{3}$ complex are shown in Fig. 1. The angle $\Theta$ and the $R(\mathrm{~N}-\mathrm{Ar})$ distance are defined in the same manner as in Ref. 4 . For clarity of presentation, positive values of $\Theta$ correspond to position of the Ar between two $\mathrm{H}$ atoms as pictured in Fig. 1. The Ar lies in a plane encompassing the $C_{3}$ axis and a $\mathrm{N}-\mathrm{H}$ bond on $\mathrm{NH}_{3}$ when $\Theta<0$. The scan of the potential energy surface varies $\Theta$ between $-180^{\circ}$ and $180^{\circ}$ with an increment of $20^{\circ} ; R$ ranges from 3.0 to $4.0 \AA$ in $0.25 \AA$ steps. Around the minimum energy orientation with $\Theta=80.0^{\circ}$, additional calculations were done for $R=4.5$ and $5.0 \AA$.

Calculated energetics are presented in Table II and Figs. 2 and 3 for $R=3.75 \AA$. Figure 2 shows clearly that the ani-

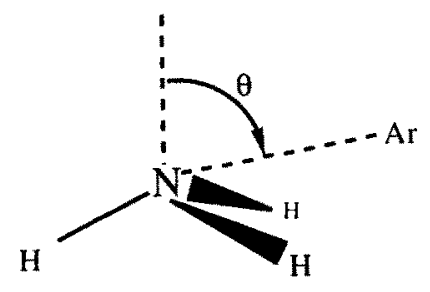

FIG. 1. Definition of the coordinate system for $\mathrm{Ar}-\mathrm{NH}_{3} . R$ refers to the $\mathrm{N}-\mathrm{Ar}$ distance and $(-)$ to the angle between the $\mathrm{N}-\mathrm{Ar}$ vector and the $z$ axis (shown as a broken line) which is collinear with the $C_{3}$ axis of $\mathrm{NH}_{3}$.

sotropy of the SCF potential is determined principally by the first-order exchange repulsion term which is the most orientation dependent. The curve representing the first-order electrostatic interaction looks very much like a mirror image of the exchange curve, albeit substantially flatter. This is due to the fact that both terms are overlap dependent. When the latter two terms are summed together, the Heitler-London curve in Fig. 2 hence retains the general shape of the exchange term. Combination with the relatively flat SCF deformation energy leaves the entire SCF interaction little changed from the $\Delta E^{\mathrm{HL}}$ curve.

The behaviors of the various post-SCF terms are illustrated in Fig. 3. It is important to note first that the anisotropy of the dispersion energy, the major contributor to $\Delta E^{(2)}$, is reciprocal to that of the exchange energy $\epsilon_{\text {exch }}^{(10)}$ (cf. Fig. 2), i.e., the minima in one nearly coincide with the maxima in the other. However, this behavior is not fully reflected in $\Delta E^{(2)}$. The $\Delta E_{\text {exch }}^{(2)}$ term which represents the difference between $\Delta E^{(2)}$ and dispersion terms [Eq. (9)] has the same general shape as the first-order exchange contribution. It has thus a "smoothing" effect on the anisotropy of $\epsilon_{\text {disp }}^{(20)}$, making its extrema less pronounced and shifting it higher in energy when proceeding from $\epsilon_{\text {disp }}^{(20)}$ to $\Delta E^{(2)}$.

$\Delta E_{\text {exch }}^{(2)}$, also shown in Fig. 3, is obtained from its parent term $\Delta E_{\text {exch }}^{(2)}$ by subtracting the $\epsilon_{\text {elst }}^{(12)}$ term [Eq. (8)]. $\Delta E_{\text {exch }}^{(2)}$ has its maxima slightly reinforced in comparison to $\Delta E_{\text {exch }}^{(2)}$, but both minima and maxima occur in the same positions. Except for the region of strong repulsion (from $-60^{\circ}$ to $\left.-160^{\circ}\right) \Delta E_{\text {exch }}^{(2)}$ amounts to nearly $1 / 2$ to $2 / 3$ of $\Delta E^{\mathrm{SCF}}$. From the close similarity of $\Delta E_{\text {exch }}^{(2)}$ and $\Delta E_{\text {exch }}^{(2)}$ curves, we may conclude that in the absence of multipole

TABLE I. Finite-field calculations of electric properties of $\mathrm{Ar}$ and $\mathrm{NH}_{3}$. All values are in atomic units.

\begin{tabular}{|c|c|c|c|c|c|c|c|c|}
\hline \multirow[b]{3}{*}{ Basis } & \multirow{2}{*}{\multicolumn{2}{|c|}{$\frac{\mathrm{Ar}}{\alpha^{n}}$}} & \multicolumn{6}{|c|}{$\mathrm{NH}_{3}$} \\
\hline & & & \multicolumn{2}{|c|}{$\mu^{\mathrm{b}}$} & \multicolumn{2}{|c|}{$\alpha_{\|}^{c}$} & \multicolumn{2}{|c|}{$\alpha_{1}^{d}$} \\
\hline & $s p d$ & $s p d f$ & $s p d$ & $s p d f$ & spd & $s p d f$ & $s p d$ & $s p d f$ \\
\hline SCF & 9.57 & 9.57 & 0.6368 & 0.6364 & 13.29 & 13.30 & 12.77 & 12.77 \\
\hline (2) & 0.20 & 0.41 & -0.036 & -0.036 & 2.44 & 2.51 & 1.02 & 1.06 \\
\hline (3) & 0.01 & 0.00 & 0.004 & 0.004 & -0.80 & -0.81 & -0.34 & -0.38 \\
\hline (4) & 0.06 & 0.09 & -0.014 & -0.014 & 0.74 & 0.75 & 0.33 & 0.34 \\
\hline $\mathrm{MP4}^{c}$ & 9.85 & 10.07 & 0.590 & 0.590 & 15.67 & 15.75 & 13.78 & 13.80 \\
\hline
\end{tabular}

a Best MBPT: 11.33 (Ref. 28); experiment: 11.06 (Ref. 29).

'Other MBPT: 0.5898 (Ref. 27); experiment: 0.5789 (Ref. 30).

'Other MBPT: 15.66 (Ref. 27).

${ }^{\mathrm{d}}$ Other MBPT: 13.73 (Ref. 27); experiment: $\alpha=14.56, \Delta \alpha=1.94$, quoted after Ref. 27 ,

${ }^{\mathrm{C}} \mathrm{MP4}=\mathrm{SCF}+(2)+(3)+(4)$. 
TABLE II. $\Theta$ dependence of interaction energy terms for the Ar- $\mathrm{NH}_{3}$ complex at $R=3.75 \AA$ (for definitions see text). All values in $\mu \mathrm{H}$.

\begin{tabular}{|c|c|c|c|c|c|c|c|c|}
\hline$\Theta$ & $\Delta E^{\mathrm{SCF}}$ & $\Delta E^{(2)}$ & $\epsilon_{\mathrm{elst}}^{(10)}$ & $\epsilon_{\mathrm{exch}}^{(\mathrm{ftos})}$ & $\epsilon_{\mathrm{disp}}^{(20)}$ & $\epsilon_{\text {elst }}^{(12)}$ & $\epsilon_{\mathrm{eis} t, \varepsilon}^{(12)}$ & $\mathrm{SCF}+\mathrm{D}^{\mathrm{a}}$ \\
\hline-180 & 705.7 & -647.9 & -405.1 & 1164.4 & -991.8 & -137.1 & -208.9 & -286.1 \\
\hline-160 & 756.7 & -689.5 & -418.6 & 1247.7 & -1028.8 & -131.6 & -198.3 & -272.2 \\
\hline-140 & 914.1 & -816.8 & -462.6 & 1527.3 & -1145.1 & -116.4 & -168.3 & -231.0 \\
\hline-120 & 1020.3 & -953.9 & -463.5 & 1730.4 & -1238.5 & -87.0 & -115.7 & -218.2 \\
\hline-100 & 861.0 & -952.4 & -360.2 & 1447.6 & -1157.0 & -50.8 & -60.8 & -296.0 \\
\hline-80 & 566.1 & -814.1 & -245.3 & 942.1 & -958.2 & -41.1 & -50.2 & -392.1 \\
\hline-60 & 389.3 & -673.0 & -218.0 & 703.4 & -813.1 & -55.8 & -75.2 & -423.8 \\
\hline-40 & 351.9 & -598.1 & -255.2 & 733.0 & -763.5 & -74.1 & -103.1 & -411.6 \\
\hline-20 & 368.8 & -573.1 & -299.8 & 835.1 & -761.3 & -86.8 & -120.9 & -392.5 \\
\hline 0 & 380.9 & -569.0 & -318.5 & 883.2 & -765.4 & -91.6 & -127.2 & -384.5 \\
\hline 20 & 364.8 & -571.5 & -298.6 & 830.0 & -759.0 & -86.6 & -120.6 & -394.2 \\
\hline 40 & 317.0 & -583.5 & -245.2 & 686.6 & -743.0 & -72.9 & -101.6 & -426.0 \\
\hline 60 & 261.0 & -614.2 & -177.7 & 518.1 & -733.0 & -53.2 & -72.6 & -472.0 \\
\hline 80 & 252.5 & -667.2 & -132.9 & 437.8 & -757.0 & -33.2 & -42.8 & -504.5 \\
\hline 100 & 345.5 & -727.1 & -155.3 & 551.9 & -832.2 & -29.5 & -37.8 & -486.7 \\
\hline 120 & 522.0 & -755.5 & -249.4 & 835.3 & -931.9 & -55.5 & -78.1 & -409.9 \\
\hline 140 & 673.5 & -729.7 & -352.6 & 1095.4 & -998.5 & -98.0 & -144.7 & -325.1 \\
\hline 160 & 718.5 & -676.6 & -400.9 & 1180.7 & -1005.7 & -128.4 & -194.1 & -287.2 \\
\hline 180 & 705.7 & -647.9 & -405.1 & 1164.4 & -991.8 & -137.1 & -208.9 & -286.1 \\
\hline
\end{tabular}

$\Delta E^{\mathrm{SCF}}+\epsilon_{\mathrm{disp}}^{(20)}$

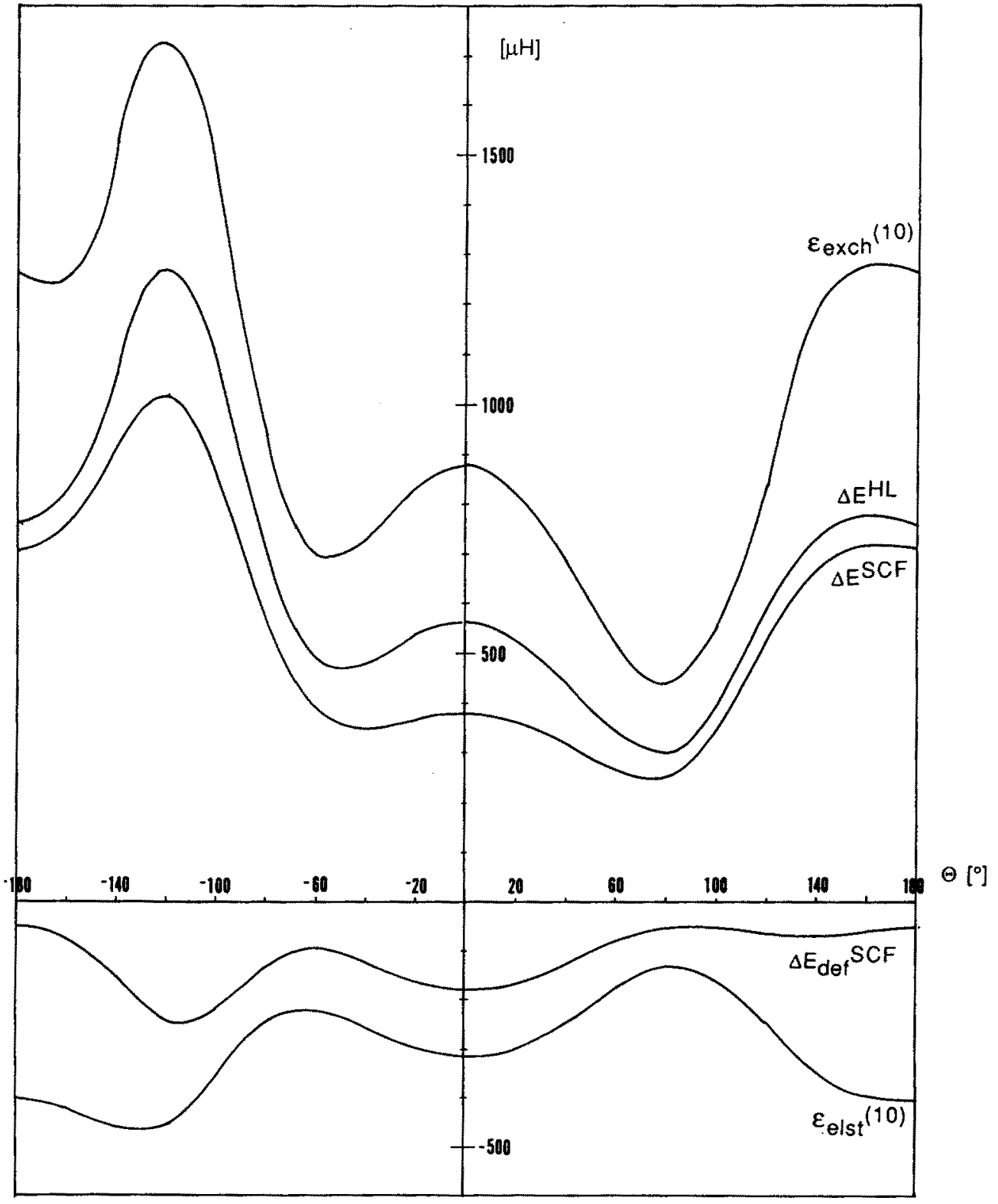

FIG. 2. $\Theta$ dependence of $\mathrm{Ar}-\mathrm{NH}_{3}$ interaction energy terms which belong to $\Delta E^{\text {SCF }}$ (for definitions, see the text). $R$ is kept at $3.75 \AA$. 


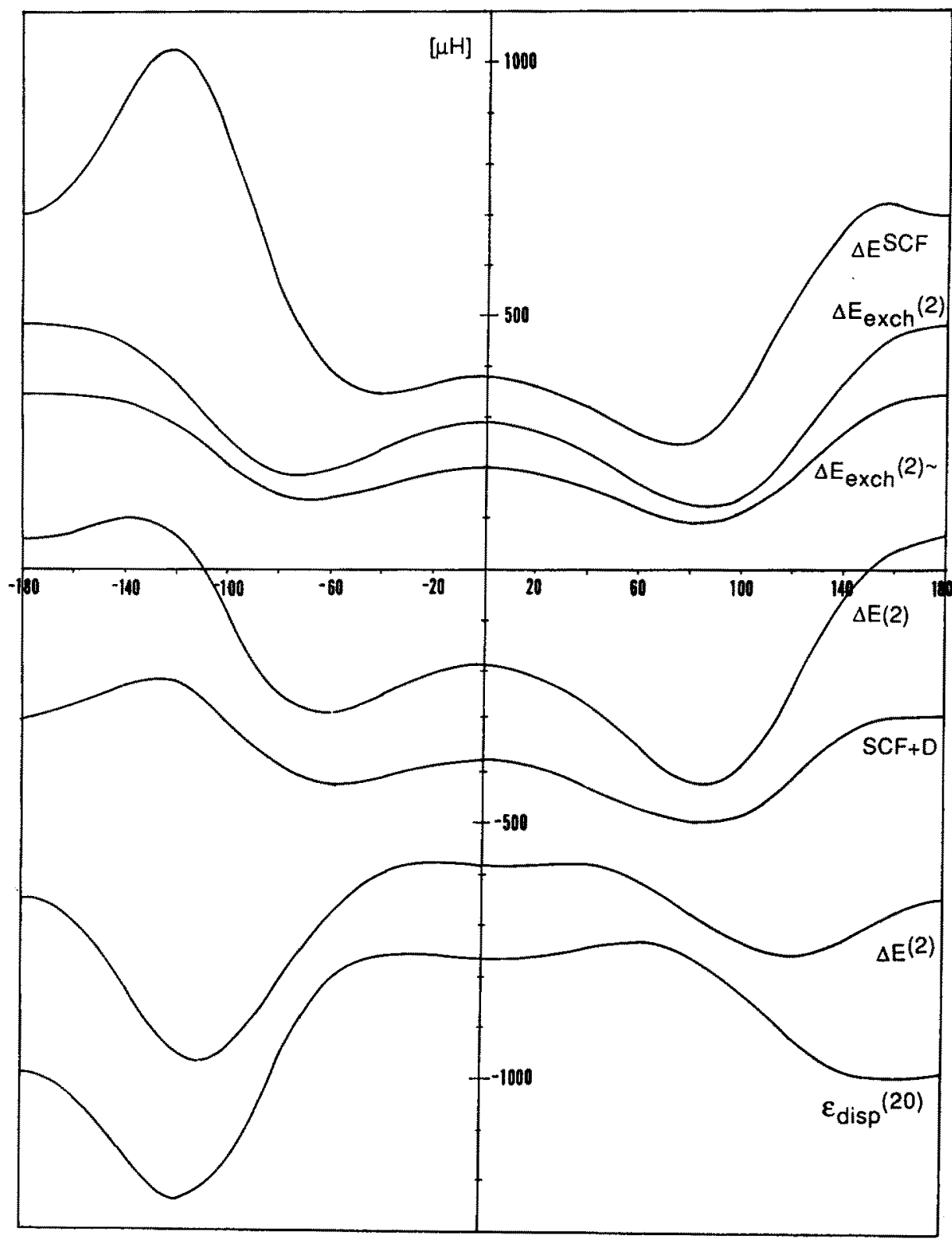

FIG. 3. $\Theta$ dependence of $\mathrm{Ar}-\mathrm{NH}_{3}$ interaction energy terms involving correlation (for definitions, see the text). $R$ is kept at $3.75 \AA$. electrostatics, as is the case of $\mathrm{Ar}-\mathrm{NH}_{3}, \Delta E_{\text {exch }}^{(2)}$ seems to be a reasonable approximation to the second-order exchange effect.

Calculated values of $\epsilon_{\text {elst }}^{(12)}$ are presented in Table II. $\epsilon_{\text {elst }}^{(12)}$ is not very large, roughly of the same order of magnitude as the SCF deformation energy. Just as the uncorrelated electrostatic term $\epsilon_{\text {elst }}^{(10)}$ (see Table II), it shows preference for regions of better overlap of $\mathrm{Ar}$ and $\mathrm{NH}_{3}$ charge distributions. For example, there is a shallow minimum around $\Theta=0^{\circ}$ (the $\mathbf{N}$ lone-pair region) as well as the more pronounced one observed for $\Theta=180^{\circ}$, i.e., where Ar overlaps with the three $\mathbf{N}-\mathbf{H}$ charge distributions simultaneously.

When this study had already been completed it was shown ${ }^{10(b, c)}$ that $\epsilon_{\text {elst }}^{(12)}$, which enters Eq. 7, should allow for additional "response" terms with respect to the original definition of Jeziorski et al. ${ }^{10(a)}$ The values of $\epsilon_{\text {elst }}^{(12)}$ with response terms, $\epsilon_{\text {elst, },}^{(12)}$, are also given in Table II. The contribution from these terms is not negligible but the above discussed properties of the electrostatic correlation term remain unaffected.

Comparison of the energetics for positive and negative values of $\Theta$ (negative $\Theta$ corresponds to Ar "eclipsing" an N$\mathrm{H}$ bond) reveals that there are two competing factors which ultimately determine the structure of this complex. Dispersion prefers the structure with $\Theta=-120.0^{\circ}$, i.e., when the Ar charge distribution best overlaps with that around a $\mathrm{N}-\mathrm{H}$ bond. On the other hand, such a structure is strongly disfavored by $\epsilon_{\text {exch }}^{(10)}$, thus also by $\Delta E^{\mathrm{SCF}}$. The latter prefers a geometry with $\Theta$ around $+80.0^{\circ}$, i.e., when Ar approaches between two $\mathrm{N}-\mathrm{H}$ bonds and the overlap between the Ar and $\mathbf{N}-\mathbf{H}$ (or $\mathbf{N}$ lone pair) charge distributions is minimal. This SCF-minimized structure coincides almost exactly with the global minimum as indicated by $\Delta E(2)$, the sum of $\Delta E^{\text {SCF }}$ and $\Delta E^{(2)}$. In other words, the anisotropy of the dispersion is not strong enough to counter the much greater angular dependence of the exchange forces. 
The interaction energy of van der Waals systems is often approximated as a sum of two contributions: $\Delta E^{\mathrm{SCF}}$ and $\epsilon_{\text {disp }}^{(20)}$, i.e., by the so-called SCF $+D$ treatment. ${ }^{32}$ One may conclude from a comparison with the $\Delta E(2)$ curve in Fig. 3 that the SCF $+D$ treatment yields a surface which is too flat and $\Delta E_{\text {exch }}^{(2)}$ is clearly necessary to properly describe its anisotropy.

Further insights concerning the $\mathrm{SCF}+\mathrm{D}$ approximation can be gleaned by considering the $R$ dependence of the interaction energy (see Table III and Fig. 4). The dispersion energy increases in absolute value substantially more rapidly than does $\Delta E^{(2)}$ as $R$ decreases. As a result, comparison between the $\Delta E(2)$ and SCF $+\mathrm{D}$ curves indicates that the latter is too deep and its minimum occurs at too short a distance. Thus the SCF $+\mathrm{D}$ potential has some clear deficiencies in predicting the equilibrium geometry of a weak complex such as this.

\section{B. Comparison with $\mathrm{Ar}_{2}$}

The energy minimum for the $\mathrm{Ar}-\mathrm{NH}_{3}$ complex was located at $R=3.75 \AA, \Theta=80.0^{\circ}$. For this minimum energy orientation, interaction energies were derived up to the MP4 level. Also computed were MP2 energetics in a basis set augmented by $f$ functions on both $\mathrm{Ar}$ and $\mathrm{N}$. The results are displayed in Table IV. This table also contains the previously published data for the Ar dimer ${ }^{12}$ (as well as some newly generated results) which are included here for comparison.

Qualitatively and quantitatively very similar energetics patterns are observed for $\mathrm{Ar}-\mathrm{NH}_{3}$ and $(\mathrm{Ar})_{2}$. First of all, $\Delta E^{\mathrm{SCF}}$ provides in both cases the dominant repulsive contribution. The most important attractive contribution arises from the $\Delta E^{(2)}$ term. The third-order contribution $\Delta E^{(3)}$ is much smaller in magnitude than $\Delta E^{(2)}$ and in both cases is repulsive. $\Delta E^{(4)}$ is slightly smaller still and is attractive in both cases. The contributions of single, double, and quadruple excitations are all repulsive in the fourth order of MPPT; the most important contribution which determines the sign and magnitude of the $\Delta E^{(4)}$ correction hence comes from triple excitations. Finally, in both complexes the inclusion of $f$ functions has very little influence upon the SCF interaction, but leads to a $100 \mu$ hartree increase in the magnitude of

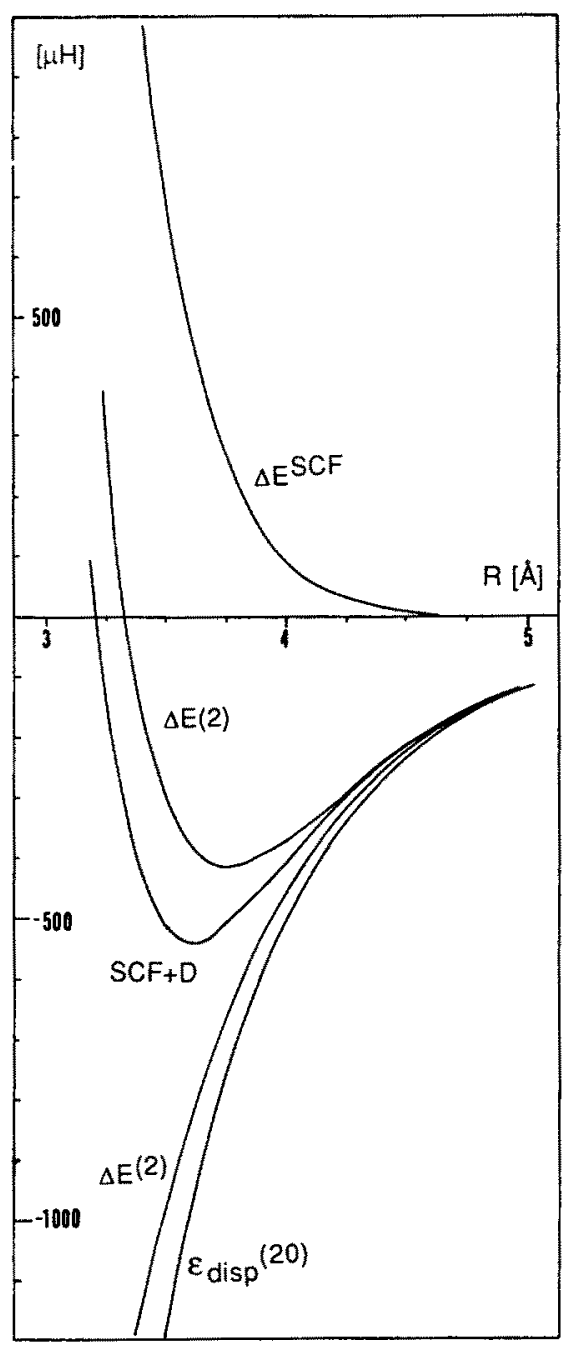

FIG. 4. $R$ dependence of interaction energy terms (for definition, see the text). $\Theta$ is kept at $80^{\circ}$.

$\Delta E^{(2)}$. This greater attraction is no doubt due to improvement in the $\epsilon_{\text {disp }}^{(20)}$ term.

There are of course some significant differences between the two systems. Due to the polarity of $\mathrm{NH}_{3}$ and its associated, more efficient perturbation of the charge cloud of $\mathrm{Ar}$, the deformation term $\Delta E_{\text {def }}^{\mathrm{SCF}}$ is more pronounced in $\mathrm{Ar}-\mathrm{NH}_{3}$.

TABLE III. $R$ dependence of interaction energy terms for the Ar- $\mathrm{NH}_{3}$ complex at $\Theta=80.0^{\circ}$ (for definitions, see the text). All values in $\mu$ hartree.

\begin{tabular}{rrrrrrrr}
\hline \hline$R, \AA$ & \multicolumn{1}{c}{$\Delta E^{\text {SCF }}$} & \multicolumn{1}{c}{$\Delta E^{(2)}$} & \multicolumn{1}{c}{$\epsilon_{\text {disp }}^{(20)}$} & \multicolumn{1}{c}{$\epsilon_{\text {elst }}^{(12)}$} & \multicolumn{1}{c}{$\epsilon_{\text {elst.r }}^{(12)}$} & SCF + D & \multicolumn{1}{c}{$\Delta E(2)^{\mathrm{b}}$} \\
\hline 3.25 & 1735.2 & -1442.8 & -1906.8 & -143.5 & -186.3 & -171.6 & 292.4 \\
3.50 & 672.8 & -977.9 & -1185.0 & -68.4 & -88.9 & -512.2 & -305.1 \\
3.75 & 252.5 & -667.2 & -757.0 & -33.2 & -42.8 & -504.5 & -414.6 \\
4.00 & 90.0 & -459.0 & -496.8 & -16.6 & -21.1 & -406.8 & -369.1 \\
4.50 & 6.8 & -225.2 & -230.8 & -4.9 & -5.8 & -224.0 & -218.4 \\
5.00 & -2.4 & -117.2 & -117.4 & -1.9 & -2.0 & -119.8 & -119.6 \\
\hline \hline
\end{tabular}

${ }^{\mathrm{a}} \Delta E^{\mathrm{SCF}}+\epsilon_{\mathrm{disp}}^{(20)}$.

${ }^{\mathrm{b}} \Delta E(2)=\Delta E^{\mathrm{SCF}}+\Delta E^{(2)}$ 
TABLE IV. Interaction energy contributions to $\mathrm{Ar}-\mathrm{NH}_{3}$ and $\mathrm{Ar}_{2}$ in the minimum (for definitions, see the text). All values are in $\mu$ hartree.

\begin{tabular}{|c|c|c|c|c|c|c|}
\hline \multirow[b]{3}{*}{ Basis } & \multicolumn{4}{|c|}{$\mathrm{Ar}-\mathrm{NH}_{3}\left(R=3.75 \AA, \Theta=80^{\circ}\right)$} & & \\
\hline & \multicolumn{2}{|c|}{ spd } & \multicolumn{2}{|c|}{$\operatorname{spdf}$} & \multicolumn{2}{|c|}{$\mathrm{Ar}_{2}(R=3.97 \AA)$} \\
\hline & & BSSE $_{\text {uncor }}$ & & BSSE $_{\text {uncor }}$ & spd & $s p d f$ \\
\hline$\overline{\Delta E^{\mathrm{SCF}}}$ & +252.5 & -39.8 & +248.4 & -72.1 & 196.9 & 196.6 \\
\hline$\Delta E^{(2)}$ & -667.2 & -1344.2 & -770.8 & -1659.4 & -448.0 & -557.2 \\
\hline$\Delta E^{(3)}$ & +73.5 & +63.2 & & & +85.4 & +86.0 \\
\hline $\mathrm{DQ}$ & +36.5 & +56.3 & & & +12.6 & +18.2 \\
\hline SDQ & +13.4 & +36.5 & & & +3.4 & +3.1 \\
\hline$\Delta E^{(4)}$ & -68.4 & -88.9 & & & -39.4 & -63.7 \\
\hline$\Delta E(n)$ & $-409.6^{a}$ & $-1409.7^{a}$ & $-522.4^{b}$ & $-1731.5^{b}$ & -205.1 & -338.3 \\
\hline$\epsilon_{\text {exch }}^{(10)}$ & 437.8 & 432.1 & & & 291.1 & \\
\hline$\epsilon_{\mathrm{ejst}}^{(10)}$ & -132.9 & -137.0 & & & -83.1 & \\
\hline$\Delta E_{\text {def }}^{\mathrm{SCF}}$ & -52.4 & & & & -11.1 & \\
\hline$\epsilon_{\text {ind }}^{(20)}$ & -126.4 & & & & -84.6 & \\
\hline$\epsilon_{\text {elst }}^{(12)}$ & -33.2 & & & & -17.4 & \\
\hline$\epsilon_{\text {disp }}^{(20)}$ & -757.0 & & & & -499.9 & \\
\hline$\Delta E_{\text {exch }}^{(2)}$ & +123.0 & & & & 69.3 & \\
\hline
\end{tabular}

Values when $n=4$.

b Values when $n=2$.

The greater polarizability of $\mathrm{NH}_{3} \mathrm{vs}$ Ar increases the dispersion attraction in the latter complex, making the $\Delta E^{(2)}$ term more negative.

The nature of $\Delta E_{\mathrm{def}}^{\mathrm{SCF}}$ warrants a more detailed analysis. As pointed out earlier, the major component of deformation is expected to arise from the second-order induction effect $\epsilon_{\text {ind }}^{(20)}$. Comparison of the latter term with $\Delta E_{\text {def }}^{\mathrm{SCF}}$ in both systems indicates that the $\epsilon_{\text {ind }}^{(20)}$ term is more than twice the magnitude of $\Delta E_{\text {def }}^{\mathbf{S C F}}$, indicating that exchange effects play a major role at the equilibrium distance. Indeed, as shown by Gutowski and Piela, the induction effect is very strongly coupled with exchange. ${ }^{17}$ On the other hand, when recomputed at the SCF minimum which occurs at the longer distance of $R=5.0 \AA\left(\Theta=80.0^{\circ}\right), \Delta E_{\text {def }}^{\mathrm{SCF}}$ is almost precisely equal to $\epsilon_{\text {ind }}^{(20)}(-4.2 \mathrm{vs}-4.5 \mu$ hartree) as exchange becomes progressively less important. In fact, according to Sadlej, $\Delta E_{\text {def }}^{\text {sCF }}$ asymptotically approaches the CHF induction $\epsilon_{\text {ind }}^{\mathrm{CHF}}$ when $R$ goes to infinity. ${ }^{16}$

The second-order exchange effect is here approximated by $\Delta E_{\text {exch }}^{(2)}$ as defined in $\mathrm{Eq}$. (8). In the equilibrium configuration, this term amounts to $+123.0 \mu$ hartree which equals $28 \%$ of the first-order exchange effect $\epsilon_{\text {exch }}^{(10)},-16 \%$ of $\epsilon_{\text {disp }}^{(20)}$, and $-30 \%$ of the total $\Delta E(4)$. In the Ar dimer, the percentage comparison of $\Delta E_{\text {exch }}^{(2)}$ is very similar: $23 \%$ of $\epsilon_{\mathrm{exch}}^{(10)}$, $-14 \%$ of $\epsilon_{\text {disp }}^{(20)}$, and $-28.0 \%$ of $\Delta E(4)$. (For the sake of comparison, in $\mathrm{He}_{2} \Delta E_{\text {exch }}^{(2)}$ amounts to $-8 \%$ of $\epsilon_{\mathrm{disp}}^{(20)}$ and $-12 \%$ of the total interaction energy. $\left.{ }^{11}\right)$ Thus, the secondorder exchange effect is very important in determining the depth of the potential minimum. As pointed out earlier in this paper, the neglect of this term may lead to an incorrect position of the minimum as well.

It is now possible to estimate the bond energy for the $\mathrm{Ar}-\mathrm{NH}_{3}$ complex. For the $\mathrm{Ar}_{2}$ complex, calculations with the $[7 s, 4 p, 2 d, 1 f]$ basis set at the MP2 and MP4 levels result- ed in bond energies of 360.6 and $338.3 \mu$ hartree, ${ }^{12}$ respectively, amounting to $80 \%$ and $75 \%$ of the exact depth of the potential minimum. Taking into account the very similar convergence pattern for MPPT in $\mathrm{Ar}_{2}$ and $\mathrm{Ar}-\mathrm{NH}_{3}$ (see above), it is concluded that the accuracy in the two should be comparable. Consequently, one may assume that our spdf MP2 value of $522.4 \mu$ hartree $=115 \mathrm{~cm}^{-1}$ represents a lower bound to the bond energy of this complex. It is interesting that our lower bound lies slightly above the experimental estimate of $102.3 \mathrm{~cm}^{-1}$ arrived at by Klemperer and coworkers. $^{3}$

Although all the calculations reported here were performed in dimer centered basis sets and are thus free from basis set superposition effects, it may, however, be instructive to analyze the error that would arise if the monomer centered basis were used. The values shown in Table IV in the BSSE $_{\text {uncor }}$ column represent results derived within the MCBS treatment. As may be noted by comparison with the preceding column, the BSSE is quite large, comparable to $\Delta E^{\mathrm{SCF}}$ and $\Delta E^{(2)}$ themselves. These errors induce a spurious minimum in the repulsive part of the SCF interaction energy curve and artificially deepen $\Delta E^{(2)}$ by a factor of 2. BSSE is less pronounced in the third and fourth orders, conforming to prior observation. ${ }^{12}$ As noticed a number of times before, upon enlargement of the basis set (by $f$ functions), the BSSE increases simultaneously with an improvement of the interaction energy. ${ }^{22,23}$ Thus, the magnitude of BSSE is not necessarily any indication of the quality of the interaction energy, contrary to some allusions in the literature. ${ }^{34,35}$

The MCBS treatment can also be applied to the perturbation terms $\epsilon_{\text {elst }}^{(10)}$ and $\epsilon_{\text {exch }}^{(10)}$ and indeed such values are

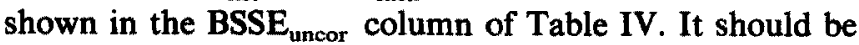
mentioned that the MCBS $\epsilon_{\mathrm{elst}}^{(10)}$ and $\epsilon_{\mathrm{exch}}^{(10)}$ terms correspond to the electrostatic and exchange effects in the Morokuma 
partitioning scheme. ${ }^{36}$ There is strong evidence that the proper description of the exchange phenomenon requires the use of dimer basis sets. ${ }^{7,14,15}$ Nevertheless, the difference between DCBS and MCBS treatments of the exchange term is very small, $1.3 \%$. However, the DCBS treatment of electrostatics does introduce certain unphysical effects. For example, the charge distribution of the Ar atom evaluated in the $\mathrm{Ar}-\mathrm{NH}_{3}$ basis is no longer spherically symmetric, leading to the appearance of a spurious multipole contribution to electrostatics. Such effects, dubbed secondary BSSE by Sadlej and Karlstrom, are also implicitly present in the supermolecular interaction energies. ${ }^{37}$ It is thus important to compare the values of $\epsilon_{\mathrm{elst}}^{(10)}$ evaluated in both dimer and monomer basis sets. The data in Table IV indicate that the DCBS and MCBS treatments of $\epsilon_{\text {elst }}^{(10)}$ differ by only $3 \%$, with the difference probably attributable to the spurious multipole electrostatics. This is in agreement with previous results for $\mathrm{He}_{2}$ and $\mathrm{HeLi}^{+}$systems, ${ }^{20}$ where no serious distortions in $\epsilon_{\text {elst }}^{(10)}$ due to DCBS were found in extended basis sets. Overall, the Heitler-London energy is underestimated by the MCBS treatment by about $10 \mu$ hartree or $3 \%$.

\section{Comparison with $\mathrm{Ar}-\mathrm{PH}_{3}$}

Supermolecular MP2 calculations were recently performed for the Ar- $\mathrm{PH}_{3}$ complex by Latajka and Scheiner. ${ }^{4}$ Their basis set for the Ar atom was composed of the welltempered sp set augmented by two $d$ functions: exponents 0.83635 and 0.33253 . To analyze the quality of their results, this basis set was tested here in $\mathrm{Ar}_{2}$. These tests produced an SCF interaction energy nearly identical to that derived with our basis set. While the SCF BSSE computed with Latajka and Scheiner's basis set was considerably smaller, $\Delta E^{(2)}$ was seriously underestimated (about $13 \%$ with respect to our value, or $18 \%$ when their $s p$ set was augmented by our $d$ functions). These observations suggest that the LatajkaScheiner $s p$ set was very good (thus the repulsive part of the potential was very reliable), but the $d$ exponents were not optimal for description of dispersion energy, the dominant attractive contribution. This contention is supported by the fact that Latajka and Scheiner's SCF dipole polarizability for Ar was equal to 8.16 a.u. (in their better basis set II), while our value is 9.57 a.u. One may thus conclude that the bond energy of $\mathrm{Ar}-\mathrm{PH}_{3}$ was underestimated in the study of Latajka and Scheiner, but due to the correct description of $\Delta E^{\mathrm{SCF}}$, the main direction sensitive contribution, their equilibrium orientation is probably accurate.

Both complexes have nearly identical geometrical configurations with the $\Theta$ angle equal to $75^{\circ}$ in $\mathrm{Ar}-\mathrm{PH}_{3}$ and $80^{\circ}$ in $\mathrm{Ar}-\mathrm{NH}_{3}$. The former appears weaker when comparing the values of $\Delta E(2)$ in the minimum $(-331 \mu$ hartree for $\mathrm{Ar}-\mathrm{PH}_{3}$ vs $-415 \mu$ hartree for $\mathrm{Ar}-\mathrm{NH}_{3}$ here). One possible factor in this difference is the much stronger $\mathrm{SCF}$ repulsion in the $\mathrm{Ar}-\mathrm{PH}_{3}$ system ( 776 vs $253 \mu$ hartree in the $\mathrm{NH}_{3}$ complex ). On the other hand, the dispersion attraction is expected to be much stronger in the complex with $\mathrm{PH}_{3}$ due to its polarizability which is twice as large as that of $\mathrm{NH}_{3}$ (SCF mean polarizabilities are $\alpha_{\mathrm{PH}_{3}}=26.7$ a.u. $\mathrm{vs} \alpha_{\mathrm{NH}_{3}}=12.9$ a.u. ). Consequently, $\Delta E^{(2)}$, which is dominated by the dispersion interaction, amounts to about $-1100 \mu$ hartree in $\mathrm{Ar}-\mathrm{PH}_{3}$, while our value for $\mathrm{Ar}-\mathrm{NH}_{3}$ is $-667.2 \mu$ hartree. Due to the abovementioned serious deficiency of the Ar basis set used in calculations of the $\mathrm{Ar}-\mathrm{PH}_{3}$ complex, the value of $\Delta E^{(2)}$ is underestimated. One may expect a roughly $13 \%$ $18 \%$ increase in this quantity upon a better choice of $d$ exponents on Ar (based upon the comparison of computed dipole polarizabilities and $\mathrm{Ar}_{2}$ data). Even a $13 \%$ increase would be sufficient to make $\mathrm{Ar}-\mathrm{PH}_{3}$ stronger than $\mathrm{Ar}-\mathrm{NH}_{3}$.

\section{ACKNOWLEDGMENTS}

This work was supported by the National Institutes of Health (GM36912) and by the Polish Academy of Sciences (CPBP01.12). Allocations of Cray time by the National Center for Supercomputer Applications at the University of Illinois and the San Diego Supercomputer Center are acknowledged. We are thankful to Dr. M. Gutowski for reading and commenting on the manuscript.

'P. Hobza and R. Zahradník, Intermolecular Complexes. The role of van der Waals Systems in Physical Chemistry and in the Biodisciplines (Academia, Prague, 1988); Structure and Dynamics of Weakly Bound Molecular Complexes, edited by H. Weber (Reidel, Dordrecht, 1987); P. Hobza and R. Zahradnik, Topic Curr. Chem. 93, 53 (1980).

${ }^{2}$ J. A. Shea and E. J. Campbell, J. Chem. Phys. 79, 4724 (1983).

${ }^{3}$ D. D. Nelson, G. T. Fraser, K. I. Peterson, K. Zhao, W. Klemperer, F. J. Lovas, and R. D. Suenram, J. Chem. Phys. 85, 5512 (1986); G. T. Fraser, D. D. Nelson, A. Charo, and W. Klemperer, ibid. 82, 2535 (1985).

${ }^{4} Z$. Latajka and S. Scheiner, J. Mol. Struct. Theochem. 198, 205 (1989). ${ }^{5}$ M. M. Szczéśniak, G. Chalasiński, Chem. Phys. Lett. 161, 5321 (1989). ${ }^{6}$ R. J. Bartlett and D. H. Silver, Int. J. Quantum Chem. Symp. 9, 183 (1975); J. S. Binkley and J. A. Pople, ibid. 9, 227 (1975); J. A. Pople, J. S Binkley, and R. Seeger, Int. J. Quantum Chem. Symp. 10, 1 (1976).

${ }^{7}$ G. Chalasiński and M. Gutowski, Chem. Rev. 88, 943 (1988); P. Hobza and R. Zahradnik, ibid. 88, 871 (1988).

${ }^{8}$ B. Jeziorski and W. Kolos, in Molecular Interactions, edited by H. Ratajczak and W. J. Orville-Thomas (Wiley, Chichester, 1982), Vol. 3.

${ }^{9}$ K. Szalewicz and B. Jeziorski, Mol. Phys. 38, 191 (1979); S. Rybak, K. Szalewicz, B. Jeziorski, and M. Jaszuński, J. Chem. Phys. 86, 5652 (1987).

${ }^{10}$ (a) B. Jeziorski, R. Moszyński, S. Rybak, and K. Szalewicz, in ManyBody Methods in Quantum Chemistry, edited by U. Kaldor (Springer, New York, 1989), p. 65; (b) R. Moszyński, S. Rybak, S. M. Cybulski, K. Szalewicz, and G. Chalasiński, Chem. Phys. Lett. (submitted); (c) S. M. Cybulski, G. Chalasiński, and R. Moszyński, J. Chem. Phys. (in press). "G. Chalasiński and M. M. Szczȩśniak, Mol. Phys. 63, 205 (1988).

${ }^{12}$ G. Chalasiński, D. J. Funk, J. Simons, and W. H. Breckenridge, J. Chem. Phys. 87, 3569 (1987).

${ }^{13}$ B. Jeziorski, M. Bulski, and L. Piela, Int. J. Quantum Chem. 10, 281 (1976).

${ }^{14}$ M. Gutowski, G. Chalasiński, and J. van Duijneveldt-van de Rijdt, Int. J. Quantum Chem. 26, 971 (1984); W. A. Sokalski, S. Roszak, P. C. Hariharan, and J. J. Kaufman, ibid. 23, 847 (1983); G. Alagona, C. Ghio, R. Cammi, and J. Tomasi, in Molecules in Physics, Chemistry, and Biology, edited by J. Maruani (Kluwer Academic, Dordrecht, 1988) vol. 2, p. 507; J. H. van Lenthe, J. G. C. M. van Duijneveldt-van de Rijdt, and F. B. van Duijneveldt, Adv. Chem. Phys. 69, 521 (1987).

${ }^{15} \mathrm{G}$. Chalasiński and M. Gutowski, Mol. Phys. 54, 1173 (1985)

${ }^{16}$ A. J. Sadlej, Mol. Phys. 39, 1249 (1980).

${ }^{17}$ M. Gutowski, and L. Piela, Mol. Phys. 64, 337 (1988).

${ }^{18}$ B. Jeziorski and M. van Hemert, Mol. Phys. 31, 713 (1976).

${ }^{19}$ S. F. Boys and F. Bernardi, Mol. Phys. 19, 553 (1970).

${ }^{20}$ M. Gutowski, F. B. van Duijneveldt, G. Chalasiński, and L. Piela, Mol. Phys. 61, 233 (1987). 
${ }^{21}$ M. Gutowski, F. B. van Duijneveldt, G. Chalasiński, and L. Piela, Chem. Phys. Letts. 129, 325 (1986).

${ }^{22}$ M. Gutowski, J. H. van Lenthe, J. Verbeek, F. B. van Duijneveldt, and G. Chalasiński, Chem. Phys. Lett. 124, 370 (1986).

${ }^{23}$ M. M. Szczȩśniak and S. Scheiner, J. Chem. Phys. 84, 6328 (1986).

${ }^{24}$ J. R. Collins and G. A. Gallup, Chem. Phys. Lett. 123, 56 (1986).

${ }^{25}$ A. J. Sadlej, Coll. Czech. Chem. Commun. 53, 1995 (1988).

${ }^{26}$ M. M. Szczéśniak, S. M. Cybulski, R. Brenstein, and S. Scheiner, J. Phys. Chem. (in press); G. Chalasiński, S. M. Cybulski, M. M. Szczȩśniak, and S. Scheiner J. Chem. Phys. (in press).

${ }^{27}$ A. J. Sadlej, Mol. Phys. 57, 509 (1986).

${ }^{28}$ I. Cernusak, G. H. F. Diercksen, and A. J. Sadlej, Chem. Phys. Lett. 128, 18 (1986).

${ }^{29}$ H. B. Levine and G. Birnbaum, J. Chem. Phys. 55, 2914 (1971).

${ }^{30}$ M. D. Marshall and J. S. Muenter, J. Mol. Spectrosc. 85, 322 (1981).

${ }^{31}$ Gaussian 86, M. J. Frisch, J. S. Binkley, H. B. Schlegel, K. Raghavachari,
C. F. Melius, R. L. Martin, J. J. P. Stewart, F. W. Bobrowicz, C. M. Rohlfing, L. R. Kahn, D. J. DeFrees, R. Seeger, R. A. Whiteside, D. J. Fox, E. M. Fluder, and J. A. Pople, Carnegie-Mellon Quantum Chemistry Publishing Unit, Pittsburgh, PA, 1984.

${ }^{32}$ C. Douketis, J. M. Hutson, B. J. Orr, and G. Scoles, Mol. Phys. 52, 763 (1984); A. Palma, S. Green, D. J. DeFrees, and A. D. McLean, J. Chem. Phys. 89, 1401 (1988).

${ }^{33}$ K. Szalewicz, S. J. Cole, W. Kolos, and R. J. Bartlett, J. Chem. Phys. 89, 3662 (1988).

${ }^{34}$ G. H. F. Diercksen and A. J. Sadlej, Mol. Phys. 59, 889 (1986); G. H. F. Diercksen, V. Kellö, and A. J. Sadlej, Chem. Phys. 96, 59 (1985).

${ }^{35} \mathrm{Z}$. Latajka and S. Scheiner, J. Comput. Chem. 8, 663 (1987).

${ }^{36} \mathrm{~K}$. Morokuma and K. Kitaura, in Molecular Interactions, edited by $\mathbf{H}$. Ratajczak and W. J. Orville-Thomas (Wiley, Chichester, 1982), Vol. 1 and references therein.

${ }^{37}$ G. Karlström and A. J. Sadlej, Theor. Chim. Acta 61, 1 (1982), 